\title{
Extraocular Cutaneous Sebaceous Carcinoma
}

National Cancer Institute

\section{Source}

National Cancer Institute. Extraocular Cutaneous Sebaceous Carcinoma. NCI Thesaurus. Code C43341.

A rare variant of sebaceous carcinoma that usually arises in the head and neck region but does not affect the ocular region. 